\title{
ON THE ABSTRACT CHARACTERIZATION OF OPERATOR SPACES
}

\author{
EDWARD G. EFFROS AND ZHONG-JIN RUAN \\ (Communicated by Palle E. T. Jorgensen)
}

\begin{abstract}
A direct proof is given for the matricial norm characterization of operator spaces.
\end{abstract}

\section{INTRODUCTION}

The abstract characterization given for linear spaces of bounded Hilbert space operators ("operator spaces") in terms of "matricially normed spaces" [9] implies that quotients, mapping spaces, and various tensor products of operator spaces may again be regarded as operator spaces. Owing in part to this result, the theory of operator spaces is having an increasingly significant effect on operator algebra theory (see, e.g., $[3,4,7])$.

The proof given in [9] appealed to the theory of ordered operator spaces [1]. In this note we will show that one can give a purely metric proof of this important theorem by using a technique of Pisier [8] and Haagerup [5] (as modified in [2]).

\section{THE MATRICIAL CHARACTERIZATION THEOREM}

Given Hilbert spaces $H$ and $K$, the linear space $B(H, K)$ of bounded operators $b: H \rightarrow K$ comes equipped with the usual norm. It follows that any operator space $V \subseteq B(H)$ is a normed vector space. However, due to the fact that a matrix of operators is again an operator, operator spaces inherit a richer "matricial norm structure", which distinguishes them from normed vector spaces. To be specific, we may regard an $m \times n$ matrix $b=\left[b_{i j}\right]$ of operators $b_{i j} \in B(H)$ as an operator from $H^{n}$ to $H^{m}$, where

$$
H^{n}=\overbrace{H \oplus \cdots \oplus H}^{n} .
$$

As a result, we may identify the space $\mathbf{M}_{m, n}(V)$ of $m \times n$ matrices over $V$ with a subspace of $B\left(H^{n}, H^{m}\right)$. This provides a distinguished norm on the matrix space $\mathbf{M}_{m, n}(V)$. The appropriate morphisms in the category of operator spaces

Received by the editors March 2, 1992.

1991 Mathematics Subject Classification. Primary 46L05.

Key words and phrases. Operator space.

The authors were partially supported by NSF. 
are those that are well behaved with respect to all of these matrix norms (see below).

Given a vector space $V$, we let $\mathbf{M}_{m, n}(V)$ denote the vector space of $m \times$ $n$ matrices $\mathbf{v}=\left[v_{i j}\right] \quad\left(v_{i j} \in V\right)$, and we let $\mathbf{M}_{n}(V)=\mathbf{M}_{n, n}(V), \mathbf{M}_{m, n}=$ $\mathbf{M}_{m, n}(\mathbb{C})$, and $\mathbf{M}_{n}=\mathbf{M}_{n, n}$. If we systematically keep track of the indices, we may use the usual identifications $\mathbf{M}_{m, n}\left(\mathbf{M}_{p, q}\right)=\mathbf{M}_{m p, n q}$, i.e., we may "delete internal brackets". Given $\mathbf{v} \in \mathbf{M}_{m, n}(V)$, $\mathbf{w} \in \mathbf{M}_{p, q}(V)$, and scalar matrices $\alpha \in \mathbf{M}_{p, m}, \beta \in \mathbf{M}_{n, q}$, we define matrices $\mathbf{v} \oplus \mathbf{w} \in \mathbf{M}_{m+p, n+q}(V)$ and $\alpha \mathbf{v} \beta \in \mathbf{M}_{p, q}(V)$ by

$$
\mathbf{v} \oplus \mathbf{w}=\left[\begin{array}{cc}
\mathbf{v} & 0 \\
0 & \mathbf{w}
\end{array}\right] \quad \text { and } \quad \alpha \mathbf{v} \beta=\left[\sum_{i j} \alpha_{k i} v_{i j} \beta_{j l}\right] .
$$

Letting $\mathbb{C}^{n}$ have the usual Hilbert space norm, we have that $\mathbf{M}_{m, n}=$ $B\left(\mathbb{C}^{n}, \mathbb{C}^{m}\right)$ is an operator space. From elementary operator considerations, the matricial norms on an operator space $V$ are connected by the relations

$$
\begin{gathered}
\|\mathbf{v} \oplus \mathbf{w}\|=\max \{\|\mathbf{v}\|,\|\mathbf{w}\|\} \quad\left(\mathbf{v} \in \mathbf{M}_{m, n}(V), \mathbf{w} \in \mathbf{M}_{p, q}(V)\right), \\
\|\alpha \mathbf{v} \beta\| \leq\|\alpha\|\|\mathbf{v}\|\|\beta\| \quad\left(\mathbf{v} \in \mathbf{M}_{m, n}(V), \quad \alpha \in \mathbf{M}_{p, m}, \quad \beta \in \mathbf{M}_{n, q}\right) .
\end{gathered}
$$

An $L^{\infty}$-matricially normed space is a vector space $V$ with norms given on each matrix space $\mathbf{M}_{m, n}(V)$ satisfying (M1) and (M2). Given two such spaces $V$ and $W$, a linear map $\varphi: V \rightarrow W$ determines a linear map

$$
\varphi^{(m, n)}: \mathbf{M}_{m, n}(V) \rightarrow \mathbf{M}_{m, n}(W):\left[v_{i j}\right] \mapsto\left[\varphi\left(v_{i j}\right)\right],
$$

and we let $\varphi^{(n)}=\varphi^{(n, n)}$. $\varphi$ is said to be completely bounded (resp. completely contractive, completely isometric) if it satisfies

$$
\|\varphi\|_{c b}=\sup \left\{\left\|\varphi^{(n)}\right\|: n \in \mathbb{N}\right\}=\sup \left\{\left\|\varphi^{(m, n)}\right\|: m, n \in \mathbb{N}\right\}<\infty
$$

(resp. $\|\varphi\|_{c b} \leq 1, \varphi^{(n)}$ is isometric for each $n \in \mathbb{N}$ ).

Our object is to prove

Theorem A. Any $L^{\infty}$-matricially normed space $V$ is completely isometric to an operator space.

Theorem $\mathrm{A}$ is a consequence of the following completely bounded analogue of the Hahn-Banach Theorem.

Theorem B. Given an $L^{\infty}$-matricially normed space $V$ and an element $\mathbf{v} \in$ $\mathbf{M}_{n}(V)$, there exists a complete contraction $\varphi: V \rightarrow \mathbf{M}_{n}$ such that $\left\|\varphi^{(n)}(\mathbf{v})\right\|=$ $\|\mathbf{v}\|$.

To see how Theorem A follows from Theorem B, let $\mathscr{S}_{n}$ be the collection of all complete contractions $\varphi: V \rightarrow \mathbf{M}_{n}$. The mapping

$$
\Phi: V \rightarrow \bigoplus_{n \in \mathbb{N}} \bigoplus_{\varphi \in \mathscr{S}_{n}} \mathbf{M}_{n}: v \mapsto(\varphi(v))
$$

is trivially completely contractive, and from Theorem B it is completely isometric.

Given $\mathbf{v} \in \mathbf{M}_{n}(V)$, the classical Hahn-Banach Theorem implies that there is an element $F \in \mathbf{M}_{n}(V)^{*}$ such that $|F(\mathbf{v})|=\|\mathbf{v}\|$. Thus Theorem B follows from 
Theorem C. Suppose that $F \in \mathbf{M}_{n}(V)^{*}$ is a contraction. Then there exists a completely contractive map $\varphi: V \rightarrow \mathbf{M}_{n}$ and contractive $1 \times n^{2}$ and $n^{2} \times 1$ matrices $\gamma$ and $\delta$ such that

$$
F(\mathbf{v})=\gamma \varphi^{(n)}(\mathbf{v}) \delta,
$$

for all $\mathbf{v} \in \mathbf{M}_{n}(V)$.

Proof. We begin by proving that there exist states $p_{0}$ and $q_{0}$ on $\mathbf{M}_{n}$ such that

$$
|F(\alpha \mathbf{v} \beta)| \leq p_{0}\left(\alpha \alpha^{*}\right)^{1 / 2}\|\mathbf{v}\| q_{0}\left(\beta^{*} \beta\right)^{1 / 2}
$$

for any matrices $\alpha \in \mathbf{M}_{n p}, \mathbf{v}=\left[v_{i j}\right] \in \mathbf{M}_{p}(V)$, and $\beta \in \mathbf{M}_{p n}$. It suffices to find $p_{0}$ and $q_{0}$ such that

$$
|F(\alpha \mathbf{v} \beta)| \leq \frac{1}{2}\left(p_{0}\left(\alpha \alpha^{*}\right)+q_{0}\left(\beta^{*} \beta\right)\right)
$$

for all such $\alpha$ and $\beta$ and all $\mathbf{v}$ with $\|\mathbf{v}\|=1$, since then for all $t>0$

$$
|F(\alpha \mathbf{v} \beta)|=\left|F\left(t^{1 / 2} \alpha \mathbf{v} t^{-1 / 2} \beta\right)\right| \leq \frac{1}{2}\left(t p_{0}\left(\alpha \alpha^{*}\right)+t^{-1} q_{0}\left(\beta^{*} \beta\right)\right),
$$

and the result follows by minimizing the right-hand side. In turn it suffices to find $p_{0}$ and $q_{0}$ such that

$$
\operatorname{Re} F(\alpha \mathbf{v} \beta) \leq \frac{1}{2}\left(p_{0}\left(\alpha \alpha^{*}\right)+q_{0}\left(\beta^{*} \beta\right)\right),
$$

since then choosing $\theta \in[0,2 \pi]$ with $e^{i \theta} F(\alpha \mathbf{v} \beta)=|F(\alpha \mathbf{v} \beta)|$, it follows that

$$
|F(\alpha \mathbf{v} \beta)|=F\left(e^{i \theta} \alpha \mathbf{v} \beta\right) \leq \frac{1}{2}\left(p_{0}\left(\alpha \alpha^{*}\right)+q_{0}\left(\beta^{*} \beta\right)\right) .
$$

We will define $p_{0}$ and $q_{0}$ to be the barycenters of measures on the state space $S$ of the $C^{*}$-algebra $\mathbf{M}_{n}$.

We let $C(S \times S)$ be the real continuous functions on $S \times S$, and let $\mathscr{E} \subseteq$ $C(S \times S)$ denote the functions of the form

$$
e_{\alpha, \mathbf{v}, \beta}(p, q)=p\left(\alpha \alpha^{*}\right)+q\left(\beta^{*} \beta\right)-2 \operatorname{Re} F(\alpha \mathbf{v} \beta)
$$

with $\|\mathbf{v}\|=1$. This is a cone because it is obvious that $\alpha \varepsilon \subseteq \varepsilon$ for $\alpha \geq 0$ and

$$
\begin{aligned}
& \left(e_{\alpha, \mathbf{v}, \beta}+e_{\alpha^{\prime}, \mathbf{v}^{\prime}, \beta^{\prime}}\right)(p, q) \\
& \quad=p\left(\left[\alpha \alpha^{\prime}\right]\left[\alpha \alpha^{\prime}\right]^{*}\right)+q\left(\left[\begin{array}{c}
\beta \\
\beta^{\prime}
\end{array}\right]^{*}\left[\begin{array}{c}
\beta \\
\beta^{\prime}
\end{array}\right]\right)-2 \operatorname{Re} F\left(\left[\alpha \alpha^{\prime}\right]\left(\mathbf{v} \oplus \mathbf{v}^{\prime}\right)\left[\begin{array}{c}
\beta \\
\beta^{\prime}
\end{array}\right]\right) \\
& \quad=e_{\alpha^{\prime \prime}, \mathbf{v}^{\prime \prime}, \beta^{\prime \prime}}(p, q),
\end{aligned}
$$

where

$$
\alpha^{\prime \prime}=\left[\alpha \alpha^{\prime}\right], \quad \mathbf{v}^{\prime \prime}=\mathbf{v} \oplus \mathbf{v}^{\prime}, \quad \beta^{\prime \prime}=\left[\begin{array}{c}
\beta \\
\beta^{\prime}
\end{array}\right],
$$

and $\left\|\mathbf{v}^{\prime \prime}\right\|=1$ by condition (M1). The function $e_{\alpha, \mathbf{v}, \beta}$ cannot be strictly negative since choosing $p_{1}, q_{1} \in S$ with $p_{1}\left(\alpha \alpha^{*}\right)=\left\|\alpha \alpha^{*}\right\|, q_{1}\left(\beta^{*} \beta\right)=\left\|\beta^{*} \beta\right\|$, it follows that

$$
\begin{aligned}
& e_{\alpha, \mathbf{v}, \beta}\left(p_{1}, q_{1}\right)=p_{1}\left(\alpha \alpha^{*}\right)+q_{1}\left(\beta^{*} \beta\right)-2 \operatorname{Re} F(\alpha \mathbf{v} \beta) \\
& \quad \geq\left\|\alpha \alpha^{*}\right\|+\left\|\beta^{*} \beta\right\|-2|F(\alpha \mathbf{v} \beta)|=2\left(\frac{1}{2}\left(\|\alpha\|^{2}+\|\beta\|^{2}\right)-|F(\alpha \mathbf{v} \beta)|\right) \\
& \quad \geq 2(\|\alpha\|\|\mathbf{v}\|\|\beta\|-|F(\alpha \mathbf{v} \beta)|) \geq 0 .
\end{aligned}
$$


Thus letting $\mathscr{K}$ be the cone of strictly negative functions on $S \times S$, we have $\mathscr{E} \cap \mathscr{K}=\varnothing$. Since $\mathscr{K}$ has interior, we have from the geometric form of the classical Hahn-Banach Theorem a measure $0 \neq \mu \in C(S \times S)^{*}$ such that $\left.\mu\right|_{\mathscr{E}} \geq 0$ and $\left.\mu\right|_{\mathscr{K}} \leq 0$. From the second inequality we conclude that $\mu$ is a positive measure, and we may assume that $\mu$ is a probability measure. Letting $p_{0}=\iint p d \mu(p, q)$ and $q_{0}=\iint q d \mu(p, q)$, i.e., taking the barycenters of the projections of the probability measures on the first and second coordinates, we have from the first inequality that

$$
0 \leq \iint e_{\alpha, \mathbf{v}, \beta}(p, q) d \mu(p, q)=p_{0}\left(\alpha \alpha^{*}\right)+q_{0}\left(\beta^{*} \beta\right)-2 \operatorname{Re} F(\alpha \mathbf{v} \beta)
$$

for any $\alpha, \mathbf{v}, \beta$ as above, and thus we have (1).

Letting $\pi$ be the standard representation of $\mathbf{M}_{n}$, i.e., the representation

$$
\pi(a)=\overbrace{a \oplus \cdots \oplus a}^{n}
$$

on $\left(\mathbb{C}^{n}\right)^{n}=\mathbb{C}^{n^{2}}$, there exist unit vectors $\xi$ and $\eta$ in $\mathbb{C}^{n^{2}}$ with $p_{0}(a)=\pi(a) \xi \cdot \xi$ and $q_{0}(a)=\pi(a) \eta \cdot \eta$ for $a \in \mathbf{M}_{n}$ (this well-known fact follows easily from [6, 4.6.18(ii)]). We let $K$ and $L$ be the subspaces of all vectors of the form $\pi(\alpha)^{*} \xi \quad\left(\alpha \in \mathbf{M}_{n, 1}\right)$ and $\pi(\beta) \eta\left(\beta \in \mathbf{M}_{1, n}\right)$, respectively. Defining $\theta: \mathbb{C}^{n} \rightarrow$ $\left(\mathbb{C}^{n}\right)^{n}=\mathbb{C}^{n^{2}}$ by

$$
\theta\left(\alpha_{1}, \ldots, \alpha_{n}\right)=\left(\left(\alpha_{1}, 0, \ldots, 0\right),\left(\alpha_{2}, 0, \ldots, 0\right), \ldots,\left(\alpha_{n}, 0, \ldots, 0\right)\right),
$$

we have that $K$ and $L$ are subspaces of $\theta\left(\mathbb{C}^{n}\right)$. We define a sesquilinear form $\langle,\rangle_{v}$ on $L \times K$ by

$$
\left\langle\pi(\beta) \eta, \pi(\alpha)^{*} \xi\right\rangle_{v}=F(\alpha v \beta) .
$$

This is bounded by $\|v\|$ since we have that

$$
\begin{aligned}
\left|\left\langle\pi(\beta) \eta, \pi(\alpha)^{*} \xi\right\rangle_{v}\right| & \leq p_{0}\left(\alpha \alpha^{*}\right)^{1 / 2}\|v\| q_{0}\left(\beta^{*} \beta\right)^{1 / 2} \\
& =\|v\|\left\|\pi(\alpha)^{*} \xi\right\|\|\pi(\beta) \eta\| .
\end{aligned}
$$

Letting $K_{0}$ and $L_{0}$ be the subspaces of $\mathbb{C}^{n}$ with $\theta\left(K_{0}\right)=K$ and $\theta\left(L_{0}\right)=L$, we have a corresponding sesquilinear function on $L_{0} \times K_{0}$, and this determines a unique linear map $\rho(v): L_{0} \rightarrow K_{0}$ of norm $\leq\|v\|$ such that

$$
F(\alpha v \beta)=\rho(v) \theta^{*} \pi(\beta) \eta \cdot \theta^{*} \pi(\alpha)^{*} \xi .
$$

Letting $\varphi(v)=\rho(v) E$ where $E$ is the projection of $\mathbb{C}^{n}$ on $L_{0}$ and using matrix notation, we may consider $\varphi(v)$ as an element of $\mathbf{M}_{n}$. Thus regarding $\xi$ and $\eta$ as $n^{2} \times 1$ matrices,

$$
F(\alpha v \beta)=\xi^{*}(\pi(\alpha) \theta) \varphi(v)\left(\theta^{*} \pi(\beta)\right) \eta
$$


where

$$
\begin{gathered}
\theta^{*} \pi(\beta)=\left[\begin{array}{cccccc}
\beta_{1} & \beta_{2} & \cdots & 0 & 0 & \cdots \\
0 & 0 & \cdots & \beta_{1} & \beta_{2} & \cdots \\
& \cdots & & & \cdots
\end{array}\right], \\
\pi(\alpha) \theta=\left[\begin{array}{cccc}
\alpha_{1} & 0 & \cdots \\
\alpha_{2} & 0 & \cdots \\
\vdots & \vdots & \vdots \\
0 & \alpha_{1} & \cdots \\
0 & \alpha_{2} & \cdots \\
\vdots & \vdots & \vdots
\end{array}\right]
\end{gathered}
$$

are $n \times n^{2}$ and $n^{2} \times n$ matrices. It follows that if $\mathbf{v}=\left[v_{i j}\right] \in \mathbf{M}_{n}(V), f_{j}=$ $\left[0, \ldots, 1_{j}, 0, \ldots, 0\right] \in \mathbf{M}_{1, n}$, and $e_{i}=f_{i}^{*} \in \mathbf{M}_{n, 1}$ then

$$
F(\mathbf{v})=\sum F\left(e_{i} v_{i j} f_{j}\right)=\sum \xi^{*} \pi\left(e_{i}\right) \theta \varphi\left(v_{i j}\right) \theta^{*} \pi\left(f_{j}\right) \eta=\gamma \varphi^{(n)}(\mathbf{v}) \delta
$$

where $\gamma=\xi^{*}\left[\pi\left(e_{1}\right) \theta \cdots \pi\left(e_{n}\right) \theta\right]$ has norm $\leq 1$ since

$$
\left[\pi\left(e_{1}\right) \theta \cdots \pi\left(e_{n}\right) \theta\right]=\left[\left[\begin{array}{ccc}
1 & 0 & \ldots \\
0 & 0 & \ldots \\
\vdots & \vdots & \\
0 & 1 & \ldots \\
0 & 0 & \ldots \\
\vdots & \vdots &
\end{array}\right]\left[\begin{array}{ccc}
0 & 0 & \ldots \\
1 & 0 & \ldots \\
\vdots & \vdots & \\
0 & 0 & \ldots \\
0 & 1 & \ldots \\
\vdots & \vdots &
\end{array}\right] \cdots\right]
$$

has norm 1 (deleting the inner brackets, we obtain a permutation matrix, i.e., each row and each column contains precisely one " 1 "), and similarly

$$
\delta=\left[\begin{array}{c}
\theta^{*} \pi\left(f_{1}\right) \\
\vdots \\
\theta^{*} \pi\left(f_{n}\right)
\end{array}\right] \eta
$$

has norm $\leq 1$.

Finally we have that $\varphi$ is a complete contraction since given $\mathbf{v} \in \mathbf{M}_{p}(V)$ and vectors $\zeta=\left(\theta^{*} \pi\left(\alpha_{k}\right)^{*} \xi\right)$ and $\omega=\left(\theta^{*} \pi\left(\beta_{l}\right) \eta\right)$ in $\left(\mathbb{C}^{n}\right)^{p}$, and letting $\alpha$ and $\beta$ be the row and column matrices with entries $\alpha_{k} \in \mathbf{M}_{n, 1}$ and $\beta_{l} \in \mathbf{M}_{1, n}$, respectively, we have from (2) that

$$
\begin{aligned}
\left|\varphi^{(p)}(\mathbf{v}) \omega \cdot \zeta\right| & =\left|\sum \varphi\left(v_{k l}\right) \theta^{*} \pi\left(\beta_{l}\right) \eta \cdot \theta^{*} \pi\left(\alpha_{k}\right)^{*} \xi\right| \\
& =\left|\sum F\left(\alpha_{k} v_{k l} \beta_{l}\right)\right|=|F(\alpha \mathbf{v} \beta)| \leq p_{0}\left(\alpha \alpha^{*}\right)^{1 / 2} q_{0}\left(\beta^{*} \beta\right)^{1 / 2}\|\mathbf{v}\| \\
& =\left[\sum p_{0}\left(\alpha_{k} \alpha_{k}^{*}\right)\right]^{1 / 2}\left[\sum q_{0}\left(\beta_{l}^{*} \beta_{l}\right)\right]^{1 / 2}\|\mathbf{v}\| \\
& =\left[\sum\left\|\pi\left(\alpha_{k}\right)^{*} \xi\right\|^{2}\right]^{1 / 2}\left[\sum\left\|\pi\left(\beta_{l}\right) \eta\right\|^{2}\right]^{1 / 2}\|\mathbf{v}\|=\|\zeta\|\|\omega\|\|\mathbf{v}\| .
\end{aligned}
$$




\section{REFERENCES}

1. M.-D. Choi and E. Effros, Injectivity and operator spaces, J. Funct. Anal. 24 (1977), 156209.

2. E. Effros, On multilinear completely bounded module maps, Contemp. Math., vol. 62, Amer. Math. Soc., Providence, RI, 1987, pp. 479-501.

3. E. Effros and Z.-J. Ruan, On apprcximation properties for operator spaces, Internat. J. Math. 1 (1990), 163-187.

4. __ Operator convolution algebras: an approach to quantum groups (to appear).

5. U. Haagerup, Decomp. of completely bounded maps, unpublished manuscript.

6. R. Kadison and J. Ringrose, Fundamentals of the theory of operator algebras. I, Academic Press, New York, 1983.

7. E. Kirchberg, On subalgebras of the CAR algebra (to appear).

8. G. Pisier, Grothendieck's Theorem for non-commutative $C^{*}$-algebras with an appendix on Grothendieck's constants, J. Funct. Anal. 29 (1978), 397-415.

9. Z.-J. Ruan, Subspaces of $C^{*}$-algebras, J. Funct. Anal. 76 (1988), 217-230.

Department of Mathematics, University of California, Los ANgeles, California 90024 E-mail address: ege@math.ucla.edu

Department of Mathematics, University of Illinois, Urbana, Illinois 61801 E-mail address: ruan@symcom.math.uiuc.edu 